J. Klin. Endokrinol. Stoffw. 2019 · 12:141-145 https://doi.org/10.1007/s41969-019-00082-9 Online publiziert: 6. November 2019

(c) Der/die Autor(en) 2019

Christian Trummer · Birgit Ratz - Marlene Pandis · Stefan Pilz • Verena TheilerSchwetz

Klinische Abteilung für Endokrinologie und Diabetologie, Universitätsklinik für Innere Medizin, Medizinische Universität Graz, Graz, Österreich

\title{
Addison-Krise - Strategien zu Therapie und Prävention
}

\section{Merkmale der Addison-Krise}

Trotz etablierter Hormonersatzstrategien zur Therapie der chronischen Nebenniereninsuffizienz ist die Mortalität betroffener PatientInnen nach wie vor erhöht [1]. Das Auftreten einer Addison-Krise zeichnet maßgeblich für die gesteigerte Mortalität verantwortlich.

Die Addison-Krise ist ein Zustand eines akuten Cortisolmangels bedingt durch erhöhten Bedarf [2, 3]. Trigger dafür sind vor allem Gastroenterititiden, die die Aufnahme der oral zugeführten Cortisolsubstitution verhindern, Infektionskrankheiten, chirurgische Eingriffe, physischer Stress oder Schmerz, aber auch extreme psychische Belastungen oder inadäquate Medikamenteneinnahme [4]. Im Rahmen dieser Triggersituationen ist neben einem erhöhten Glukokortikoidbedarf vermutlich auch eine teils durch Inflammation verursachte "Cortisolresistenz" pathophysiologisch relevant. In einer prospektiven Untersuchung konnte von 8,3 Krisen pro 100 PatientInnenjahren berichtet werden, was impliziert, dass eine/r von 12 PatientInnen im nachfolgenden Jahr von einer potenziell lebensbedrohlichen Addison-Krise betroffen sein wird [5] eine alarmierende Zahl.

Wiewohl in der Literatur ein Konsens über die möglichen klinischen Symptome einer Addison-Krise herrscht, liegt bislang immer noch keine einheitlich akzeptierte Definition der AddisonKrise vor. Gründe dafür sind in erster Linie das Fehlen eines singulären charakteristischen Symptoms sowie die unspezifischen Beschwerden, die von PatientInnen beim Auftreten einer Ad-
dison-Krise empfunden werden. Die Endocrine Society definiert die Addison-Krise als medizinischen Notfall, der mit Hypotonie, ausgeprägten akuten Bauchschmerzen und deutlichen Laborauslenkungen einhergeht und einer unverzüglichen Behandlung bedarf [6]. Eine andere Definition beschreibt die Addison-Krise als (a) massive Einschränkung des Allgemeinbefindens mit Vorliegen von zumindest zwei der folgenden Symptome/Zeichen: Hypotonie (systolischer Blutdruck $<100 \mathrm{mmHg}$ ), Übelkeit und Erbrechen, starke Müdigkeit, Fieber, Somnolenz, Hyponatriämie ( $\leq 132 \mathrm{mmol} / \mathrm{l}$ ) oder Hyperkaliämie, Hypoglykämie und (b) parenterale Glukokortikoid-Verabreichung gefolgt von klinischer Besserung [3]. Eine pragmatische Definition wäre eine Verschlechterung des Allgemeinzustands mit absoluter Hypotonie (systolischer Blutdruck $<100 \mathrm{mmHg}$ ) oder relativer Hypotonie (systolischer Blutdruck $\geq 20 \mathrm{mmHg}$ niedriger als normalerweise), mit typischen Symptomen, die sich innerhalb von 1-2 h nach parenteraler GlukokortikoidVerabreichung deutlich bessern (d.h. deutlicher Rückgang der Hypotonie innerhalb einer Stunde und Verbesserung der klinischen Symptome innerhalb von 2h) [1].

Nicht nur die unspezifischen Symptome der Addison-Krise, sondern auch das seltene Vorkommen derselben und die dadurch fehlende Erfahrung vieler Ärztinnen und Ärzte bedingen die Tatsache, dass PatientInnen mit Addison-Krisehäufig inadäquat behandelt werden [3].

\section{Maßnahmen zur Verbesserung der Versorgung}

Die Problematik unterstreicht die Notwendigkeit der regelmäßigen und ausführlichen Schulung von PatientInnen mit primärer und sekundärer Nebenniereninsuffizienz zur Prävention einer Addison-Krise, zur Erkennung einer Krise und zum korrekten Management derselben. Da jedoch gezeigt werden konnte, dass selbst geschulte PatientInnen vorhandenes Wissen zur Dosissteigerung im Falle einer akuten Erkrankung nicht adäquat anwenden konnten [7], sollten diese Schulungen standardisiert, strukturiert und wiederholt erfolgen, am besten gemeinsam mit Familienangehörigen/Freunden [4, 7] sowie anderen Betroffenen, um einen Erfahrungsaustausch zu ermöglichen, der sich ebenso als günstig erwiesen hat [8].

PatientInnen müssen lernen, ihre Glukokortikoidsubstitution (Hydrocortison $10-12 \mathrm{mg} / \mathrm{m}^{2}$ aufgeteilt auf 2 oder 3 Dosen mit Verabreichung der Hälfte oder von 2/3 der Tagesdosis in der Früh [9]) in Situationen mit erhöhtem Bedarf adäquat zu steigern. Sie erhalten einen Notfallausweis sowie ein Notfallset (1 Ampulle Hydrocortison $100 \mathrm{mg}$, Spritze und Nadeln zur subkutanen Selbstverabreichung) mit Anleitung (siehe - Abb. 1) und einen Merkzettel zur Dosissteigerung (siehe $\bullet$ Abb. 2). Auch die praktische Anwendung des Notfallsets muss in oben genannten Schulungen regelmäßig geübt werden $[8,10]$, um eine korrekte Handhabung im Akutfall zu ermöglichen.

An der klinischen Abteilung für Endokrinologie und Diabetologie der Medizinischen Universität Graz werden Pa- 
tientInnen mit Nebenniereninsuffizienz seit März 2018 strukturiert mit einem Hydrocortison-Notfallset versorgt. Bei Ausgabe des Notfallsets werden PatientInnen und deren Angehörige vom behandelnden Endokrinologen sowie einer diplomierten Pflegeperson hinsichtlich der Anwendung geschult. Es erfolgt zusätzlich eine Aufklärung darüber, dass auch nach Verwendung des Notfallsets umgehend ärztliche Hilfe zur weiteren Therapie aufgesucht werden muss.

Insgesamt wurden bisher (Stand Juli 2019) über 80 Notfallsets an PatientInnen ausgegeben, die an einer primären oder sekundären Nebenniereninsuffizienz leiden und eine regelmäßige orale Glukokortikoidsubstitution benötigen. Soweit bekannt, verabreichten sich seitdem zumindest zwei dieser PatientInnen im Rahmen einer (drohenden) AddisonKrise selbstständig komplikationslos Hydrocortison, beide wurden anschließend in einer internistischen Notaufnahme weiterbehandelt und konnten das Krankenhaus beschwerdefrei wieder verlassen.

Das beiliegende Begleitblatt zur Glukokortikoid-Dosissteigerung und Behandlung einer (drohenden) AddisonKrise soll sowohl betroffenen PatientInnen als auch medizinischem Fachpersonal helfen, Situationen mit erhöhtem Glukokortikoidbedarf adäquat zu managen. Es basiert auf einer systematischen Literaturrecherche, welche nach internem Review der Autorenschaft dieses Manuskriptes erstellt wurde [3, 6, 11-15]. Es kann Grundlage oder Ergänzung für die unabkömmlichen, regelmäßig notwendigen Schulungen sein, die PatientInnen mit primärer oder sekundärer Nebenniereninsuffizienz benötigen, mit dem Ziel, die Mortalität Betroffener zu senken.

J. Klin. Endokrinol. Stoffw. 2019 · 12:141-145 https://doi.org/10.1007/s41969-019-00082-9 (c) Der/die Autor(en) 2019

\section{Trummer $\cdot$ B. Ratz $\cdot$ M. Pandis · S. Pilz $\cdot$ V. Theiler-Schwetz \\ Addison-Krise - Strategien zu Therapie und Prävention}

\section{Zusammenfassung}

Die chronische Nebenniereninsuffizienz ist trotz adäquater Hormonersatzstrategien nach wie vor mit einer erhöhten Mortalität assoziiert. Der Grund hierfür liegt im Auftreten von Addison-Krisen, hervorgerufen durch einen Zustand eines akuten Cortisolmangels in erster Linie durch erhöhten Bedarf (beispielsweise bei Gastroenteritis, Infektionskrankheiten, inadäquater Medikamenteneinnahme etc.). Eine pragmatische Definition der Addison-Krise ist eine Verschlechterung des Allgemeinzustands mit absoluter (systolischer Blutdruck $<100 \mathrm{mmHg}$ ) oder relativer Hypotonie (systolischer Blutdruck $\geq 20 \mathrm{mmHg}$ niedriger als normalerweise) und mit typischen Symptomen, die sich innerhalb von $1-2 \mathrm{~h}$ nach parenteraler GlukokortikoidVerabreichung deutlich verbessern. Durch die unspezifischen Symptome und das seltene Vorkommen einer Addison-Krise werden viele PatientInnen inadäquat behandelt. Regelmäßige Schulungen von PatientInnen mit primärer und sekundärer Nebenniereninsuffizienz zur Prävention einer Addison-Krise, zur Erkennung einer Krise und zum korrekten Management sind daher unerlässlich. Ziel ist das Erlernen der korrekten Hydrocortison-Dosissteigerung in Situationen mit erhöhtem Bedarf sowie der Handhabung des Notfallsets mit enthaltener Hydrocortison-Ampulle, die bei drohender Addison-Krise subkutan verabreicht werden sollte.

\section{Schlüsselwörter}

Nebenniereninsuffizienz - Cortisolmangel · Hydrocortison · Notfallset · Hypotension

\section{Adrenal crisis-strategies for therapy and prevention}

\section{Abstract}

Despite current hormone replacement schemes, chronic adrenal insufficiency is still associated with increased mortality. Reasons include the occurrence of adrenal crises, triggered by situations of acute cortisol deficiency mostly due to increased need, e.g. in cases of gastroenteritis, infections, and inadequate intake of medications. A pragmatic definition of adrenal crisis is an acute deterioration in health associated with absolute (systolic blood pressure $<100 \mathrm{mmHg}$ ) or relative hypotension (systolic blood pressure $\geq 20 \mathrm{mmHg}$ lower than usual), with features that resolve within 1 to $2 \mathrm{~h}$ after parenteral glucocorticoid administration. Due to unspecific symptoms and the rare occurrence of an adrenal crisis, many patients are still inadequately treated. Regular patient education programs are warranted for patients with primary and secondary adrenal insufficiency for the prevention, detection, and correct management of adrenal crisis. Aims include teaching patients to carry out dose adaptations in situations with increased need and to handle their emergency kit correctly, i.e. to correctly perform subcutaneous hydrocortisone injections in the case of an impending adrenal crisis.

\section{Keywords}

Adrenal insufficiency - Cortisol deficiency . Hydrocortisone - Emergency kit · Hypotension 


\section{Verabreichen der „, Notfallspritze “ zur Therapie/Verhinderung einer Addisonkrise}

Eine Nebennierenkrise bzw. Addisonkrise ist ein lebensbedrohliches Zustandsbild, welches typischerweise bei schweren Erkrankungen bzw. Infekten (vor allem Magen-Darm Erkrankungen) auftreten kann oder falls die Glukokortikoidtherapie vergessen bzw. nicht weiter eingenommen wird. Typische Beschwerden sind Übelkeit, Erbrechen, extreme Abgeschlagenheit bis Schläfrigkeit, niedriger Blutdruck und Bauchschmerzen. Zu Vermeidung oder Therapie solcher Notfallsituationen müssen Sie oder Ihre Angehörigen die „Notfallspritze“ mit Hydrocortison $100 \mathrm{mg}$ verabreichen und danach sofort ärztliche Hilfe (Transport ins Krankenhaus) aufsuchen!

Wann soll ich die Notfallspritze mit $100 \mathrm{mg}$ Hydrocortison verabreichen?

Die Notfallsprize mit $100 \mathrm{mg}$ Hydrocortison muss im Falle einer schweren Erkrankung, eines Unfalles, wiederholten Erbrechens, schweren Durchfalls oder bei sonstigen Beschwerden mit Verdacht auf Addisonkrise SOFORT verabreicht werden (auch im Zweifelsfall unbedingt

verabreichen)! Sie oder Ihre Angehörigen können die Notfallspritze verabreichen.

Wie soll ich die Notfallspritze mit $100 \mathrm{mg}$ Hydrocortison verabreichen?

In Ihrem Notfallset haben Sie 1 Ampulle $100 \mathrm{mg}$ Hydrocortison, eine $2 \mathrm{ml}$ Spritze, eine „dicke“ rote Nadel/Kanüle G18 zum Aufziehen der Lösung, eine orange dünne Nadel/Kanüle $G 25$

eine Thrombosespritze" oder Insulininjektion

Z. an der Oberschenkelaußenseite ist möglich und sogar schneller voll wirksam im Vergleich 22 Minuten), die subcutane Injektion wird aber primär empfohlen da sie in der Regel auch von den PatientInnen eindeutig bevorzugt wird.
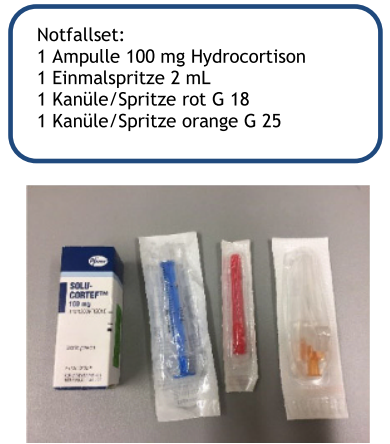

3) Entfernen Sie die kleine gelbe Schutzkappe, die sich in der Mitte

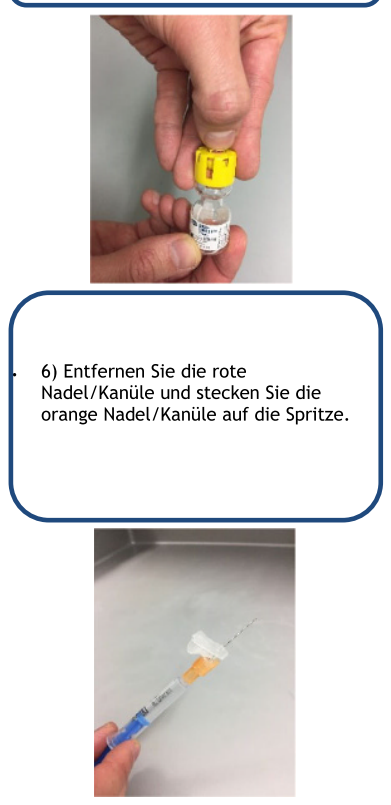

1) Nehmen Sie die Ampulle heraus und drücken Sie fest mit dem Daumen auf die gelbe Plastikkappe damit das Lösungsmittel sich mit dem Pulver ganz unten vermischen kann.

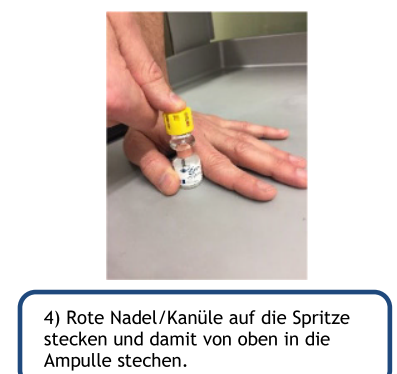

stecken und damit von auf die Spritze

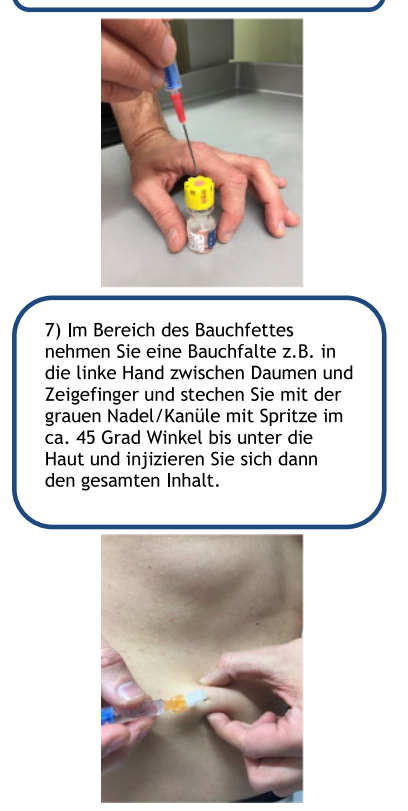

2) Ampulle ein paar Sekunden leich schütteln zum Durchmischen bis sic das Pulver aufgelost hat und eine klare Flüssigkeit entstanden ist.

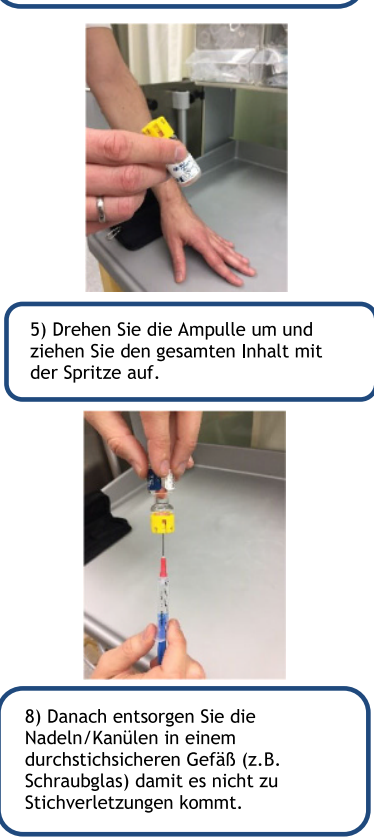

Abb. 1 Anleitung zur Verabreichung der subkutanen Hydrocortison-Injektion bei (drohender) Addison-Krise 


\section{Informationen für PatientInnen}

Da bei Ihnen krankheitsbedingt eine Produktionsstörung der Nebennierenhormone vorliegt, müssen Sie täglich lebenswichtige Glukokortikoidhormone als Medikament einnehmen. Dies ist ein Ratgeber für Sie (und Ihre Ärztin/lhren Arzt), wie Sie die Einnahme der Hormonpräparate in bestimmten Situationen durchführen sollen.

Notfallausweis, Tabletten und Notfallspritze wenn möglich immer bei sich tragen!

Niemals darf die Hormontherapie mit Glukokortikoiden (z.B. Hydrocortone) abgesetzt werden!

Bei Erkrankungen mit Arztkontakt den Notfallausweis und diesen Ratgeber vorzeigen!

\begin{tabular}{|c|c|}
\hline \multicolumn{2}{|c|}{ 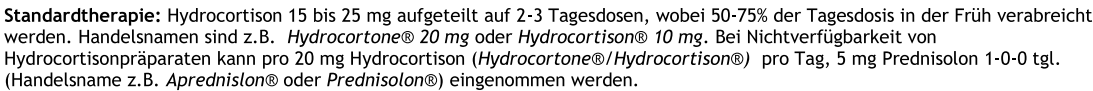 } \\
\hline $\begin{array}{l}\text { Fieberhafte Infekte/Erkrankungen } \\
\text { Bei Temperatur von } 37,5 \text { bis } 39 \text { Grad Celsius: doppelte } \\
\text { Tagesdosis (z.B. statt Hydrocortone } 20 \mathrm{mg} 1 / 2-1 / 4-1 / 4 \text { dann } \\
1-1 / 1-1 / 2) \text {. } \\
\text { Bei Temperatur über } 39 \text { Grad Celsius: dreifache } \\
\text { Tagesdosis } \\
\text { Die erhöhte Dosis soll für ca. } 2 \cdot 3 \text { Tage (d.h. bis man sich } \\
\text { schon deutlich besser fühlt) eingenommen werden; } \\
\text { danach weiter mit der Dosis wie immer. Zudem bei } \\
\text { Fieber auf ausreichende Flüssigkeitszufuhr (genügend } \\
\text { Trinken!) achten. } \\
\text { Bei anderen Erkrankungen mit Antibiotikatherapie oder } \\
\text { allgemeinen Erkrankungen bei denen man bettlägerig ist: } \\
\text { doppelte Tagesdosis bis zur Besserung. }\end{array}$ & $\begin{array}{l}\text { Durchfall (Diarrhoe) und Erbrechen } \\
\text { Bei Durchfall ist die Tagesdosis sofort zu verdoppeln; bei } \\
\text { schwerem Durchfall ist sofort die Notfallspritze mit } 100 \\
\text { mg Hydrocortison subcutan (= unter die Haut) zu } \\
\text { verabreichen und es muss sofort ärztliche Hilfe (in erster } \\
\text { Linie Transport mit der Rettung ins Krankenhaus) } \\
\text { aufgesucht werden! } \\
\text { Bei Erbrechen ist sofort die zuvor eingenommene Dosis in } \\
\text { dann doppelter Dosis einzunehmen. Sollte dann innerhalb } \\
\text { von } 30 \text { Minuten wiederholt Erbrechen auftreten ist sofort } \\
\text { die Notfallspritze mit } 100 \mathrm{mg} \text { Hydrocortison subcutan } \\
\text { (=unter die Haut) zu verabreichen und es muss sofort } \\
\text { arzztliche Hilfe (in erster Linie Transport mit der Rettung } \\
\text { ins Krankenhaus) aufgesucht werden! } \\
\text { Bei Erbrechen und/oder Durchfall auf ausreichende } \\
\text { Flüssigkeitszufuhr (genügend Trinken!) achten. }\end{array}$ \\
\hline
\end{tabular}

Stresssituationen und körperliche Anstrengungen

Bei ausgeprägten psychischen Stresssituationen wie z.B. Trauerfall oder Prüfungen kann ausnahmsweise $5 \cdot 10 \mathrm{mg}$ Hydrocortison (z.B. 30-60 Minuten vor der Prüfung) zusätzlich zur normalen Tagesdosis eingenommen werden.

Bei ausgeprägten körperlichen Belastungen wie z.B. längerem Dauerlauf/Marathon oder mehrstündiger Wanderung soll 30-60 Minuten vor der Belastung 5-10 mg Hydrocortison zusätzlich zur normalen Tagesdosis eingenommen werden.

Informationen für medizinisches Fachpersonal

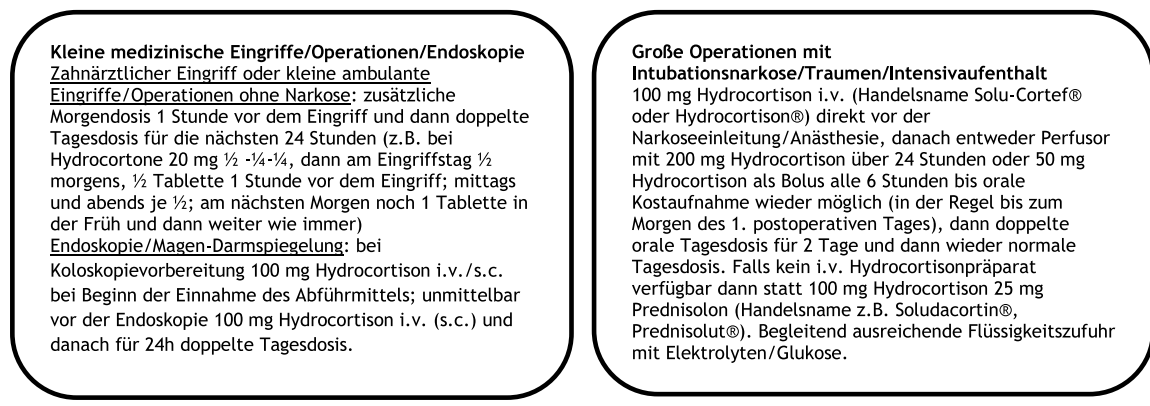

Geburt $100 \mathrm{mg}$ Hydrocortison i.v. bei Beginn der Wehen; dann Hydrocortison Perfusor 200mg/24h (oder $50 \mathrm{mg}$ Hydrocortison i.v. alle $6 \mathrm{~h}$ ) bis Geburt; dann doppelte Tagesdosis für $24 \mathrm{~h}$ und dann wie immer.

Addisonkrise

SOFORT $100 \mathrm{mg}$ Hydrocortison i.v. (Handelsname Solu-Cortef $\circledast$ oder Hydrocortison $\circledast$ ), danach entweder Perfusor mit $200 \mathrm{~m}$

Hydrocortison über 24 Stunden oder $50 \mathrm{mg}$ Hydrocortison als Bolus alle 6 Stunden. Falls kein i.v. Hydrocortisonpräparat verfügbar dann statt $100 \mathrm{mg}$ Hydrocortison $25 \mathrm{mg}$ Prednisolon (Handelsname Z.B. Soludacortin $\Theta$, Prednisolut $($ ). Bei Besserung schrittweise Dosisreduktion bzw. Umstellung auf orale Hydrocortisontherapie. SOFORT 1 Liter physiologische Kochsalzlösung ( $\mathrm{NaCl} 0,9 \%)$

innerhalb einer Stunde und danach weitere Flüssigkeit und evtl. Glukose 5\% (cave: auf mögliche Hypoglykämie achten!) je nach individueller Situation.

Literatur: J Clin Endocrinol Metab 101:364-389, 2016; Clin Med (Lond) 17:258-262, 2017; Internist 57:457-469, 2016; J Intern Med 275:104-115, 2014; Win Klin Wochenschr Educ

Abb. $2 \triangleleft$ Merkzettel für PatientInnen und medizinisches Fachpersonal zur Hydrocortison-Dosissteigerung 


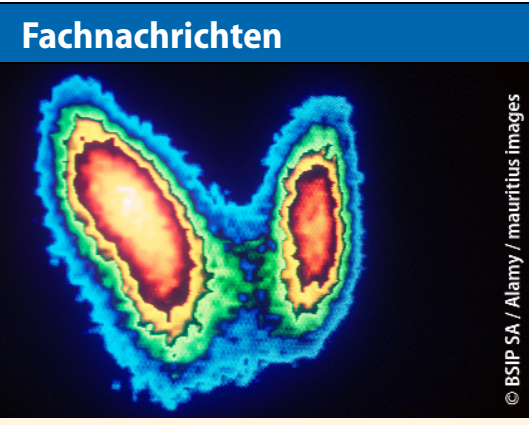

\section{Korrespondenzadresse}

DDr. Christian Trummer

Klinische Abteilung für Endokrinologie und Diabetologie, Universitätsklinik für Innere Medizin, Medizinische Universität Graz Auenbruggerplatz 15, 8036 Graz, Österreich christian.trummer@medunigraz.at

PD Dr. Verena Theiler-Schwetz, PhD Klinische Abteilung für Endokrinologie und Diabetologie, Universitätsklinik für Innere Medizin, Medizinische Universität Graz Auenbruggerplatz 15, 8036 Graz, Österreich verena.schwetz@medunigraz.at

Funding. Open access funding provided by Medical University of Graz.

\section{Einhaltung ethischer Richtlinien}

Interessenkonflikt. C. Trummer, B. Ratz, M. Pandis, S. Pilz und V. Theiler-Schwetz geben an, dass kein Interessenkonflikt besteht.

Für diesen Beitrag wurden von den Autoren keine Studien an Menschen oder Tieren durchgeführt. Für die aufgeführten Studien gelten die jeweils dort angegebenen ethischen Richtlinien.

Open Access Dieser Artikel wird unter der Creative Commons Namensnennung 4.0 International Lizenz (http://creativecommons.org/licenses/by/4.0/deed. de) veröffentlicht, welche die Nutzung, Vervielfältigung, Bearbeitung, Verbreitung und Wiedergabe in jeglichem Medium und Format erlaubt, sofern Sie den/die ursprünglichen Autor(en) und die Quelle ordnungsgemäßnennen, einen Link zur Creative Com mons Lizenz beifügen und angeben, ob Änderungen vorgenommen wurden.

\section{Literatur}

1. Rushworth RL, Torpy DJ, Falhammar H (2019) Adrenal crisis. N Engl J Med 381 (9):852-861

2. Bornstein SR, Allolio B, Arlt W, Barthel A, DonWauchope A, Hammer GD et al (2016) Diagnosis and treatment of primary adrenal insufficiency: an endocrine society clinical practice guideline. J Clin Endocrinol Metab 101(2):364-389

3. Allolio B (2015) Extensive expertise in endocrinology. Adrenal crisis. Eur JEndocrinol 172(3):R115-24

4. Puar TH, Stikkelbroeck NM, Smans LC, Zelissen PM, Hermus AR (2016) Adrenal crisis: still a deadly event in the 21 st century. Am J Med 129(3):339.e1-339.e9

5. Hahner S, Spinnler C, Fassnacht M, Burger-Stritt S, Lang K, Milovanovic D et al (2015) High incidence of adrenal crisis in educated patients with chronic adrenal insufficiency: a prospective study. J Clin Endocrinol Metab 100(2):407-416

6. Bornstein SR, Allolio B, Arlt W, Barthel A, DonWauchope A, Hammer GD et al (2016) Diagnosis and treatment of primary adrenal insufficiency: an endocrine society clinical practice guideline. J Clin Endocrinol Metab 101(2):364-389

7. Shepherd LM, Tahrani AA, Inman C, Arlt W, Carrick Sen DM (2017) Exploration of knowledge and understanding in patients with primary adrenal insufficiency: a mixed methods study. BMC Endocr Disord 17(1):47

8. Repping-Wuts HJ, Stikkelbroeck NM, Noordzij A Kerstens M, Hermus AR (2013) A glucocorticoid education group meeting: an effective strategy for improving self-management to prevent adrenal crisis. Eur JEndocrinol 169(1):17-22

9. Charmandari $E$, Nicolaides NC, Chrousos GP (2014) Adrenal insufficiency. Lancet 383(9935):2152-2167

10. White K, Arlt W (2010) Adrenal crisis in treated Addison's disease: a predictable but undermanaged event. Eur J Endocrinol 162(1):115-120

11. Pazderska A, Pearce SH (2017) Adrenal insufficiency-recognition and management. Clin Med (Lond) 17(3):258-262

12. Pulzer A, Burger-Stritt S, Hahner S (2016) Addison's disease: primary adrenal insufficiency. Internist (Berl) 57(5):457-469

13. Husebye ES, Allolio B, Arlt W, Badenhoop K, Bensing S, Betterle $C$ et al (2014) Consensus statement on the diagnosis, treatment and followup of patients with primary adrenal insufficiency. JIntern Med 275(2):104-115

14. Hahner S, Burger-Stritt S, Allolio B (2013) Subcutaneous hydrocortisone administration for emergency use in adrenal insufficiency. Eur J Endocrinol 169(2):147-154

15. Ratz BM (2018) Therapeutical and educational aspects of adults suffering from primary adrenal insufficiencya systematic review

Hinweis des Verlags. Der Verlag bleibt in Hinblick auf geografische Zuordnungen und Gebietsbezeichnungen in veröffentlichten Karten und Institutsadressen neutral.

\section{Ausschreibung des \\ Österreichischen \\ Schilddrüsenpreises 2020}

Gestiftet von der Österreichischen Schilddrüsengesellschaft und der Österreichischen Gesellschaft für Nuklearmedizin und Molekulare Bildgebung

Zur Förderung der Erforschung von Schilddrüsenerkrankungen, insbesondere der klinischen Forschung, sowie Erforschung der Methoden zur Diagnose der Schilddrüsenerkrankungen und deren Therapie, wird der Österreichische Schilddrüsenpreis 2020 ausgeschrieben.

Der Forschungspreis wird von der Österreichischen Schilddrüsengesellschaft (OSDG) und von der Österreichischen Gesellschaft für Nuklearmedizin und Molekulare Bildgebung (OGNMB) finanziert und ist mit $€ 1.000$ - dotiert.

\section{Ausschreibungsbedingungen:} https://www.schilddruesengesellschaft.at/ schilddruesenpreis

Einreichungsschluss: 31.1 .2020

\section{ÖSTERREICHISCHE SCHILDDRÜSENGESELLSCHAFT}

Austrian Thyroid Association

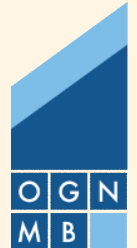

TERREICHISCHE GESELLSCHAFT FÜ NUKLEARMEDIZIN \& MOLEKULARE BILDGEBUNG 